\title{
Hemodynamic Sensor in Cardiac Implantable Electric Devices: The Endocardial Accelaration Technology
}

\author{
Stefania Sacchi, MD ${ }^{1 *}$, Danilo Contardi ${ }^{3}$, Paolo Pieragnoli, MD', \\ Giuseppe Ricciardi, $\mathrm{MD}^{\mathbf{1}}$; Andrea Giomi, $\mathrm{MD}^{\mathbf{1}}$ and Luigi Padeletti, $\mathbf{M D}^{\mathbf{1 , 2}}$ \\ ${ }^{1}$ Istituto di Clinica Medica e Cardiologia, University of Florence, Italy \\ ${ }^{2}$ Cliniche Gavazzeni, Bergamo, Italy \\ ${ }^{3}$ SORIN Group Italia S.r.l., Milano, Italy
}

Submitted January 2013. Accepted for publication July 2013.

\begin{abstract}
There have been substantial progresses in the technology of cardiac implantable electric devices (CIEDs) during the past decades. One of the progresses is represented by the development of a hemodynamic sensor embedded at the tip of a pacing lead that measures myocardial contractility by the analysis of myocardial mechanical vibrations occurring during the cardiac cycle. This sensor, providing continuous hemodynamic monitoring, could play an important role in clinical practice because of several clinical applications in CIEDs recipients. The objectives of this work are to report how this sensor operates and to review the main findings about its clinical applications.
\end{abstract}

Keywords: hemodynamic sensor, peak endocardial acceleration, myocardial contractility, cardiac implantable devices.

\section{INTRODUCTION}

Cardiac implantable electric devices, pacemakers (PMs), implantable cardioverterdefibrillators (ICDs) and devices for cardiac resynchronization therapy (CRT), represent today the "well-proven efficacious" tools in the treatment of cardiac arrhythmias and heart failure (HF) [1]. Over time, a progressive improvement of the available diagnostic and therapeutic algorithms has been observed, mainly related to the detection of cardiac arrhythmias, management of pacing/sensing thresholds, reduction of unnecessary ventricular pacing, prevention of atrial tachyarrhythmias recurrence, development of rate responsive pacing and rate adaptive atrioventricular (AV) delay functions, allowing a better management of patients. A reliable continuous hemodynamic information could improve the outcome of patients by a) continuously optimizing the device settings, b) preventing acute recurrences of heart

*Corresponding author: Stefania Sacchi, Istituto di Clinica Medica e Cardiologia, University of Florence, Viale Morgagni 85, 50134 Florence, Italy. E-mail: ste_sacchi@yahoo.it. Other authors: lpadeletti@interfree.it; danilo.contardi@sorin.com; paolopieragnoli@virgilio.it; vpric73@hotmail.com; giomimail@gmail.com. 
failure, and c) improving clinical decision-making processes [2-4]. One of the major and most recent innovations is represented by the development of a hemodynamic sensor incorporated at the tip of a pacing lead. This sensor, measuring myocardial vibrations during the cardiac cycle by an endocardial accelerometer suitable for the lead, has been developed for a direct measurement of myocardial contractility [5-10].

The objectives of this work are to report how this endocardial acceleration technology works and to review the main clinical findings about its current use.

\section{PRINCIPLE OF ENDOCARDIAL ACCELERATION TECHNOLOGY}

\subsection{Myocardial Contractility}

The contractile state of the heart is currently determined invasively by a direct recording of the maximum rate of rise of the left ventricular pressure ( $\mathrm{LV} \mathrm{dP/dt} \mathrm{max)} \mathrm{[11,} \mathrm{12].} \mathrm{LV}$ $\mathrm{dP} / \mathrm{dt} \max$ is universally accepted as a good index to assess acute changes in contractility and it is currently considered the gold-standard for the assessment of the contractile force [12].

\subsection{Peak Endocardial Acceleration (PEA) Signal as an Index of Myocardial Contractility}

The hemodynamic endocardial acceleration (EA) sensor, embedded at the tip of an implantable pacing lead, is a detector of the acceleration of the endocardial wall of the left ventricle. It has been defined as a "Microphone Inside the Heart" because it records and amplifies myocardial vibrations during the cardiac cycle transforming them into an electric signal that is processed and converted into a peak endocardial acceleration's signal (PEA signal).

Preliminary studies have shown that changes at the peak of these myocardial vibrations occurring during isovolumic systole (peak endocardial acceleration I, PEA-I) are closely related to changes in $\operatorname{LV~} \mathrm{dP} / \mathrm{dt} \max [5,7,8,10]$. Thus, these studies have identified the peak of endocardial acceleration (PEA) as an index of myocardial contractility.

The peak of the myocardial vibrations can be recorded also during the isovolumic relaxation phase of the cardiac cycle (peak endocardial acceleration II, PEA-II). Preliminary studies have shown that PEA-II closely correlates with the min LV dP/dt value $[7,8]$. Thus, PEA components' measurement, corresponding to the extent of cardiac cycle's isovolumic contraction and relaxation, allows monitoring of both systolic and diastolic function of the left ventricle.

Furthermore, Tassin et al. reported a close correlation between amplitude/timing of PEA-I and PEA-II components and amplitude/timing of first and second heart sounds, as detected by standard external phonocardiographic (PCG) recordings, supporting that PEA and heart sounds are manifestations of the same phenomena [13]. In fact, it is well known that heart sounds generation is dominated by myocardial vibrations during both isovolumic contraction and relaxation, as well as by other factors including blood acceleration/deceleration within the ventricles and the movements of valves leaflets. 


\subsection{Endocardial Acceleration Sensor}

Myocardial mechanical vibrations can be recorded by an endocardial accelerometer (EA sensor) equipped with pass-band filters tuned to reject the spurious components derived from body movements and external interferences. The pass-band filter has been selected as a result of the initial in-vivo research around the endocardial acceleration signal. The filter is a numeric pass-band filter with two first-order corner frequencies of 10 and $128 \mathrm{~Hz}$. The piezoceramic bender and the electronics within the sensor act both by filtering the acceleration components outside the range of $10 \mathrm{~Hz}$ to $128 \mathrm{~Hz}$. There is no dynamic or adaptive tuning of the filtering, hence there is no tuning algorithm. Myocardial vibrations can be effectively detected regardless of the endocardial position of the sensor [6, 7, 10]. Independent from the recording site, the endocardial acceleration signal can be equally detected and processed $[6,7,10]$. The correlation between the peak endocardial acceleration signal and the human cardiac contractility expressed in terms of $\mathrm{LV} \mathrm{dP} / \mathrm{dt}$ max exists independent of the recording site by the SonR sensor, whether it is the apex of the right ventricle, the coronary sinus or the right atrial appendage [6]. This signal provides a reliable estimation of the global cardiac contractility rather than a measure of the regional contractility or local kinetics $[6,7]$.

The interior of the sensor consists of the following three basic components (Figure 1a): 1) A "seismic mass and microspring" system, to amplify the mechanical movements due

(a)

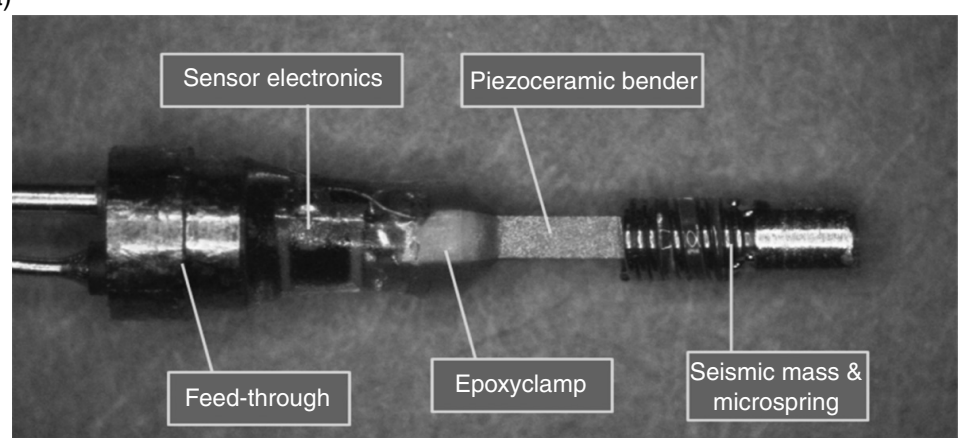

(b)
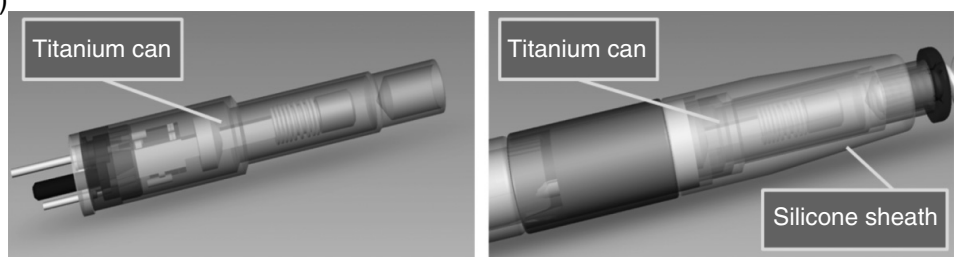

Figure 1. Endocardial accelerometer (EA) sensor. (a) Interior components. (b) Exterior components: a titanium can (left) that coils up the inner sensor components, and is covered by a silicone sheath (right). 
to myocardial vibrations and to improve the resistance to mechanical shocks. The function of the microspring is to provide a mechanical dampening to decouple the mass from the bender in case of shock. This arrangement ensures adequate resilience of the sensor to mechanical shocks, such as those that could occur during handling of the lead. 2) A "piezoceramic bender", to transduce flexural stresses into electric (voltage) signal. This voltage signal derived from the sensor to identify the PEA-I component does not interfere at all either with sensing signals or with delivered therapy. In order to detect the PEA-I component, every time an R-wave is detected by the ventricular sensing channel, the implanted device opens a "sensor detection window", just after the R-wave, for a specific limited time interval. This allows the system to completely uncouple the detection of the acceleration components from the sensing and pacing functions. The device applies commonly used blanking techniques to decouple the sensor and sensing signals. In fact, when a "sensor detection window" is opened after the R wave, the atrial pace/sense dipole is in any case in refractory conditions (a post-ventricular refractory period is triggered by any ventricular paced or sensed event, in order to avoid atrial oversensing due to the ventricular activation). This sensing control system does not impact the performance of atrial arrhythmias monitoring. 3) An "epoxy clamp", defining the free vibrating part of the bender. The "sensitivity of the sensor" is determined by the physical arrangement of its components. The seismic mass participates, together with the piezoceramic bender transducer and the overall structure of the assembly, to obtain the required sensitivity. The electronic circuit inside the sensor acts as a "buffering circuit" in order to ensure proper transmission of the signal to the device without applying any voltage gain to the signal generated by the transducer.

The assembly of the inner portion of the sensor is completed by connecting the basic components to the electronics (first-line processing and filtering of the voltage signal), which is in turn connected to a feed-through element, aiming to maintain full hermeticity and to ensure electric continuity with lead conductors. The exterior of the sensor (Figure 1b) consists of a rigid titanium can coiling-up the inner assembly to protect the basic structures of the EA sensor, which is in turn protected by an insulating silicone sheath.

\subsection{EA Signal Acquisition and Processing}

The EA signal is generated by the vibrations of the cardiac structures during the cardiac cycle. These vibrations propagate through the cardiac walls. When the vibration arrives in proximity of the sensor-carrier lead tip, the vibration itself produces a microscopic oscillation of the seismic mass, which in turn produces a flexural stress on the piezoceramic bender. The piezoceramic bender transforms the oscillation into a voltage signal. The signal is then sent to the circuits of the implanted device, which are capable of the following: 1) reading the real-time filtered signal, and 2) extracting the peak-topeak amplitudes of the PEA-I components.

\subsection{Technology of the Lead}

The described EA sensor has been designed to be sealed into the tip of a lead for endocardial permanent pacing/sensing function, making this technology suitable for clinical applications associated with implantation of cardiac implantable electric 
devices (CIEDs) with or without resynchronization capability. The advantages of an endocardial sensor installed in the distal portion of a pacing lead are the following: 1) a permanent implantation in the right heart with continuous monitoring of the EA signal that is an index of myocardial contractility [7]; 2) no need for additional leads as pressure sensor leads or conductance catheters to achieve a hemodynamic monitoring. The main limitation of this EA sensor lead, that is similar to a "standard lead" in terms of sensing, pacing functions and handling and physical characteristics, seems to be related to the fact that it works only in combination with a dedicated device, equipped with a specific atrial port designed to receive a tripolar connection of the lead. This tripolar connector is based on a design of an IS-1 BIP connection standard.

The proximal part of the EA sensor lead is depicted in Figure 2. One of the hardware poles is common to the two functions (managed via software by the CIED). The sensor is electrically connected between the distal electrode (tip) line and the dedicated (third pole) sensor line. The distal electrode line is the sensor reference (ground) pole, while the sensor line is the active pole, where the signal is transmitted. During the sensor powering periods, the current flows on the distal and sensor lines and through the sensor, activating the sensor and allowing the device to sample the EA. No current is delivered to the heart because no current path exists outside the lead during the sensor activation period. Of note, the lead portion just outside the header of the device has been designed with an inner "transition-spring" mechanical component; in the connector portion, the transition spring has the function to allow the lead to bend while protecting the conductors from being overstressed and providing pushability for the connection. In the distal end, the stiffness is reduced to improve handling and positioning in the atrium. A dual-layer silicone and polyurethane insulation ensures consistent mechanical protection and electrical insulation to the lead conductors. The electric continuity between the CIED and the EA sensor allows a bidirectional flow for the following functions: 1) Power supply from device batteries to sensor electronics (controlled injection of current pulses). The frequency of the transmission is $512 \mathrm{~Hz}$. The impact on the lifetime of the device is estimated to be less than or equal to 0.4 years. 2) EA signal transmission from sensor electronics to the device (for further

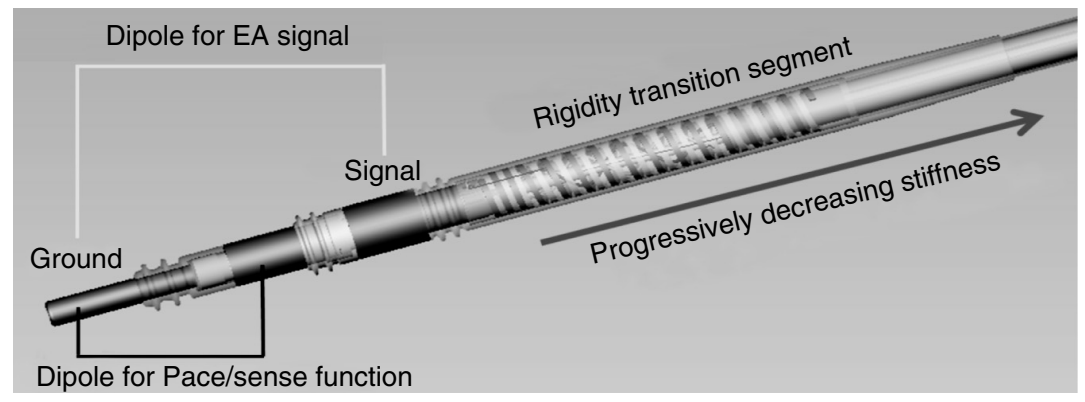

Figure 2. Proximal part of the EA sensor lead with tripolar connection: distal bipole for pacing/sensing function (based on a design of an IS-1 BIP connection standard) and dipole for EA signal transmission to the device. 
processing). The EA signal has significant information in the range between about 10 and $120 \mathrm{~Hz}$, with a peak in the $40-70 \mathrm{~Hz}$ range, and has essentially no component above $250 \mathrm{~Hz}$. The peak-to-peak amplitude of the systolic component ranges between $0.2 \mathrm{~g}\left(1 \mathrm{~g}=9.81 \mathrm{~m} / \mathrm{s}^{2}\right)$ and $2 \mathrm{~g}$, depending on several factors related to cardiac contractility, atrioventricular (AV) and interventricular (VV) timings, and more generally to the hemodynamic state of the heart. The signal of the sensor is sampled at $512 \mathrm{~Hz}$ by the device and digitized. A numeric band-shaping filtering $(10-128 \mathrm{~Hz})$ is subsequently applied to the signal, providing the rejection of spurious signal components from potential sources of interference. The appropriate filtering has been selected after extensive study of the EA signal, based on wide-band $(1-1000 \mathrm{~Hz})$ recording of the accelerometer signals in several different conditions. Beat to beat variability of the signal is largely dominated by the cardiac variability, which is greater for heart failure patients than for other patients with preserved myocardial function. In the currently available device, the algorithm robustness is ensured by averaging the measured signal over a few consecutive cycles (typically 4 to 7 ). The initial findings have shown that sensor signal is not interfered by patient's voice, cough, pulmonary rales. The heart is indeed almost "floating" inside the chest, and the propagation of external vibrations is significantly attenuated. Low-frequency phenomena, such as components related to the respiration activity, can be also cut-off. For instance, the atrial tissue depolarization in atrial fibrillation condition - considered to be a spurious component to the sensor - very rarely exceeds $10 \mathrm{~Hz}$ (corresponding to $600 \mathrm{bpm}$ ); thus this component is effectively cut-off. As the technology evolves, in terms of miniaturization and improvement of the sensitivity of the sensor, several lead models equipped with EA sensor have been released, featuring different fixation mechanisms to the endocardial walls (tined or screw-in) and a progressive downsizing of the lead body. Recently, the target chamber for sensor implantation switched from the right ventricle (RV) to the right atrium (RA). The choice to move the sensor placement from the right ventricle to the right atrium has nothing to do with the EA technology itself. The encapsulation of a micro-accelerometer sensor inside the tip of the right ventricular pacing lead represents a constraint because of ongoing technological progress and changes in the implantation indications [9]. In particular, patients suffering from heart failure are regular candidates for the implantation of a CRT-D system (cardiac resynchronization therapy with pacemaker/defibrillator function), which requires an RV lead designed to pace, sense and defibrillate with a complex array of coils and electrodes [9]. Consequently, a right atrial lead equipped with PEA sensor could be used for hemodynamic sensing of the ventricular contraction/ relaxation phases of the cardiac cycle, without impending the development of the ventricular leads $[9,10]$. This is to avoid the inclusion of the EA sensor at the tip of an RV lead, which is already jammed with defibrillation coils and multiple conductors. Moreover, Bordachar et al. demonstrated that a sensor placed in the atrium offers the major advantage of sensing a signal coincident with atrial contraction [9]. This might allow, in future applications, inclusion of this component of PEA in various algorithms, such as that for the automatic control of atrial pacing capture or AV delay optimization, with a view, among other objectives, to sharpen the tachyarrhythmia discrimination by implanted devices. 


\section{LITERATURE REVIEW}

\subsection{Rate-Responsive Pacing Function}

The close relationship between contractility and heart rate has been employed to develop a closed-loop algorithm capable of driving rate-response (RR) in pacemakers equipped with EA sensor. This sensor, measuring cardiac contractility, bases its rate response on myocardial contractility, a measurement physiologically modulated by the baroreceptors, derived directly from the heart, which changes with heart rate during exercise, although less prominently [14]. Two studies have shown that changes in PEA signal recorded by EA sensor are closely correlated with sinus node rate during exercise and pharmacological interventions $[14,15]$. In patients with normal chronotropic function, Clementy et al. first reported that PEA signal was linearly correlated with sinus rates during cycloergometer effort test [15]. In patients with chronotropic incompetence implanted with PEA-based PM, the variations in PEA-driven paced heart rate were closely correlated with those observed in controls with normal sinus rhythm [14]. Furthermore, a robust correlation was found between PEA variations and calculated energy cost of exercise (metabolic equivalent, MET) during the steps of standardized protocols (bycicle or Bruce) [16].

\subsection{Pacing for Neurally-Mediated Syncope}

The therapeutic role of permanent dual chamber pacing in patients with malignant neurally-mediated syncope (NMS) refractory to pharmacological therapy remains controversial. This is mostly due to the fact that standard pacing systems cannot detect the hypotension usually preceding bradycardia when an NMS occurs. Previous studies postulated that a reflex increase in sympathetic activity results in vigorous myocardial contractions before an NMS [17]. As a consequence, the increased inotropic activity, as detected by EA sensor during head-up tilt test, was hypothesized to allow an early intervention by pacing to avoid NMS event $[18,19]$. Deharo et al. evaluated the rate of symptomatic NMS recurrences and quality of life (QoL) score in a 6-month period preceding the PM implantation and after conventional DDI pacing (asynchronous atrioventricular pacing) or DDDR pacing (rate-responsive synchronous atrioventricular pacing) driven by contractility, in a randomized cross-over design. Contractility-driven DDDR pacing induced a higher reduction of symptomatic NMS recurrence, although QoL scores were similar in the two pacing modes [20].

\subsection{AV Delay Optimization in DDD Pacing (Synchronous Atrioventricular Pacing)}

A dedicated algorithm based on PEA signal was developed to identify the optimal AV delay (AVD) value in patients affected by complete AV-block and indication for DDD pacing [21]. The EA signal rises from the combination of two effects: the myocardial contractility and the noise due to the movements of the mitral valve. In the condition of a very short AV delay value, the effect of mitral valve becomes predominant ("big noise" generated by the truncation of the A-wave). In the condition of a long AV delay value, the effect of mitral valve becomes minor ("small noise"). The optimal AV delay corresponds to the inflection point of the EA signal curve. Significant reports showed that the optimal AVD identified by the EA algorithm, both at rest and under effort, was correlated with that identified by the gold-standard echocardiographic method [21, 22]. 


\subsection{Cardiac Resynchronization Therapy (CRT) Optimization}

Cardiac resynchronization has been demonstrated to be an efficient therapy for patients with systolic advanced HF and dyssynchrony; however, up to $40 \%$ of patients do not show improvement in left ventricular performance or clinical symptoms after CRT [23]. Device optimization is expected to lower the proportion of non-responders, and continuous changes of the programmed $\mathrm{AV}$ and $\mathrm{VV}$ intervals may be required [24]. A third-generation algorithm based on PEA signal was conceived, aiming at a continuous automatic AV and VV delays optimization in CRT devices. This algorithm determines first the optimal VV synchrony, then the optimal AV synchrony under different conditions (atrial sensing and pacing, patient at rest or under effort), globally lasting a few minutes. VV delay optimization is carried out by night, with the patient at rest. For each specific VV delay configuration, the algorithm forces an AV delay scanning in DDD pacing mode (over a range of AV delay compatible with full ventricular capture with the max value of the range defined by an AV delay shorter than the spontaneous $\mathrm{AV}$ ) and measures the EA values accordingly. The resulting "EA versus AV delay" curves are compared by measuring the EA area below the curves. The value of the EA area is an expression of the overall contractility related to a specific VV delay under different filling conditions. The optimal VV delay value corresponds to the highest value of the EA area. The therapeutic effect of an optimal VV delay was demonstrated to consist of a positive hemodynamic response to CRT defined as an increase by $10 \%$ in the invasive $\mathrm{LV} \mathrm{dP/dt} \max$ [25]. Once the optimal VV delay is determined and reprogrammed, the device looks for the optimal AV delay with the patient still at rest. As the variable electromechanical latencies and the inter and intra atrial delays may significantly affect filling performance, the algorithm identifies the optimal AV delay in both atrial sensing (DDD $50 \mathrm{bpm}$ to favour sinus rhythm) and atrial pacing conditions (DDD 70 bpm to overdrive sinus rhythm) $[4,21,23,26]$. The optimal AV delay always corresponds to the inflection point of the EA versus AV delay value curve [26]. The filling performance may also significantly change with heart rate, due to physical exercise. During the day the algorithm checks if the patient's heart rate exceeds a programmable target value for testing $(90 \mathrm{bpm}$ to $110 \mathrm{bpm}$ ). In this case the procedure of AV delay optimization is carried out at the testing heart rate to identify the optimal AV delay under effort. Every week, the algorithm iterates the full procedure to identify and reprogram the best device settings to assure optimal VV synchrony and AV synchrony in any patient condition.

The "CLEAR" multicenter pilot study [27] prospectively assessed the clinical benefit of automatic CRT optimization by EA hemodynamic sensor. The automatic CRT optimization ("PEA" group) was compared with the Standard-of-Care clinical criteria ("SoC" group) of the participating centres. The primary endpoint of the study was the Packer's Clinical Combined Criterion (all-cause mortality, HF-events, NYHA functional class, QoL score) evaluated at 1-year follow-up, focusing on the rate of clinical response (i.e patients who significantly improved according to Packer's endpoint). The study was conducted between 2005 and 2009 years at 51 Centres in 8 European Countries. The implanted system consisted of a CRT-PM with an EA MiniBest ${ }^{\circledR}$ (Sorin Biomedica, Saluggia, Italy) sensor lead in the right ventricle. The 
difference in the primary endpoint between the two groups was highly significant: $76 \%$ PEA vs. $62 \%$ SoC $(p=0.0285)$ [27].

A new trial is currently in progress to further confirm the clinical benefit of automatic CRT optimization by EA sensor [28]. This trial includes HF patients of any etiology, eligible for CRT-ICD, in sinus rhythm and NYHA functional class III and IV, with LVEF $<35 \%$ and QRS $>120 \mathrm{~ms}$. The objective of this study is to compare in a randomized experimental design (2:1 ratio) patients with ambulatory CRT optimization based on weekly re-assessment of the hemodynamic performance by EA sensor and patients optimized only once before hospital-discharge (by using standardized echocardiographic method: longest LV filling time without A-wave truncation and maximum Aortic-VTI). The same endpoint of the pilot CLEAR study (Packer's criterion) is used, imposing a double-blinded assessment of the rate of clinical response at 1-year follow-up. The target chamber for the proposed EA sensor, named SonR $®$, is the right atrium ("SonRtip" screw-in lead, SORIN CRM srl, Saluggia, Italy).

\section{DISCUSSION}

We have reviewed the main clinical findings about the EA technology. Preliminary studies have demonstrated that the measurement of myocardial contractility by an accelerometer sensor embedded at the tip of an implantable pacing lead is feasible [5-10] and it may have wide promising clinical applications [14-22, 27], as summarized in Table 1. Among them, the potential contributions of EA sensor timings in the detection of cardiac mechanical events and in the precise measurement of systolic and diastolic periods, suggest that this sensor could be useful in the monitoring of cardiac function and adaptative optimization of CRT [29].

At present, the development of CRT devices that are able to find the optimal setting automatically for both AV and VV intervals, and to reset it regularly at rest and during exercise represents an important field of research [30]. The inaccurate programming of the AV delay, VV delay, or both has proven to be a weighty cause of non-response to CRT [4, 30]. Although echocardiography is currently the most reliable method to optimize AV and VV intervals, this time-consuming, operatordependent task is not performed systematically and, when performed, is limited to conditions of rest in a supine position [4, 30]. Several alternative nonechocardiographic methods have been proposed to optimize AV and VV intervals such as invasive measurement of the LV $\mathrm{dP} / \mathrm{dt} \max$, acoustic cardiography,

Table 1. Clinical Applications of EA technology in CIEDs recipients

\begin{tabular}{l}
\hline EA sensor \\
\hline 1) Rate-Responsive Pacing Function \\
2) Pacing for Neurally-Mediated Syncope \\
3) AV Delay Optimization in DDD Pacing \\
4) CRT Optimization
\end{tabular}


impedance cardiography and methods based on automated algorithms such as QuickOpt method and SmartDelay algorithm [30]. These algorithms use intracardiac electrograms to automatically optimize AV and VV delays in biventricular pacing [4, 30]. The FREEDOM and SMART-AV studies [31, 32], that evaluated prospectively the benefit of a frequent optimization of the AV and VV intervals using the QuickOpt method and the SmartDelay algorithm respectively, did not report a significant contribution to further improve responsiveness to CRT compared with the empirical setting or the echo-doppler iterative method [4]. The automated algorithms are operator-independent and time-saving CRT optimization tools. Their lack of benefit may be due to the rest condition and to the low frequency of the AV and VV intervals optimization. Moreover, Porciani et al. have shown that automatic algorithms such as QuickOpt may fail to identify the best AV and VV intervals, since in patients with severe heart failure the electrical activation times do not always coincide with the times of mechanical activation [33]. While previous automatic algorithms did not demonstrate any beneficial effect, the automatic PEA method, based on the mechanical acceleration of the heart has been shown to be closely consistent with the cardiac ultrasound in the timings of aortic and mitral valve closures and in the estimation of systolic and diastolic intervals durations [29]. The benefit of this method could be due to the fact that it allows a weekly automatic optimization of the $\mathrm{AV}$ and VV intervals at rest and also during exercise. The CLEAR study demonstrated clinical benefits of the CRT achieved by the PEA configuration compared with CRT optimized by standard clinical procedure [27].

\section{CONCLUSIONS}

The EA technology, embedded at the tip of a standard permanent lead for cardiac pacing/sensing function has opened a new era in cardiac pacing allowing measurement of the myocardial contractility. This technology is employed in driving rate-response pacing function, preventing and treating neurally mediated syncope, and optimizing automatically AV and VV delays in CRT.

\section{CONFLICT OF INTEREST:}

Luigi Padeletti is a consultant for Biotronik, Boston, Medtronic, Sorin and St. Jude Medical.Danilo Contardi is an employee of Sorin Group Italia.

\section{REFERENCES}

[1] McMurray JJ, Adamopoulos S, Anker SD, Auricchio A, Böhm M, Dickstein K, Falk V, Filippatos G, Fonseca C, Gomez-Sanchez MA, Jaarsma T, Køber L, Lip GY, Maggioni AP, Parkhomenko A, Pieske BM, Popescu BA, Rønnevik PK, Rutten FH, Schwitter J, Seferovic P, Stepinska J, Trindade PT, Voors AA, Zannad F, Zeiher A. ESC Guidelines for the diagnosis and treatment of acute and chronic heart failure 2012: The Task Force for the Diagnosis and Treatment of Acute and Chronic Heart Failure 2012 of the European Society of Cardiology. Developed in collaboration with the Heart Failure Association (HFA) of the ESC. Eur Heart J, 2012, 33:1787-1847.

[2] Sweeney MO. Peak endocardial acceleration signals for atrial mechanical activation . J Cardiovasc Electrophysiol, 2011, 22:584-586.

[3] Kadhiresan K, Carlson G. The role of implantable sensors for management of heart failure. Stud Health Technol Inform, 2004, 108:219-227. 
[4] Antonini L, Auriti A, Pasceri V, Meo A, Pristipino C, Varveri A, Greco S, Santini M. Optimization of the atrioventricular delay in sequential and biventricular pacing: physiological bases, critical review, and new purposes. Europace, 2012, 14:929-938.

[5] Bombardini T, Gaggini G, Marcelli E, Parlapiano M, Plicchi G. Peak Endocardial Acceleration reflects heart Contractility Also in Atrial Fibrillation. Pacing Clin Electrophysiol, 2000, 23(9):1381-1385.

[6] Bongiorni MG, Soldati E, Arena G, Quirino G, Vernazza F, Bernasconi A, Garberoglio B. Is local myocardial contractility related to endocardial acceleration signals detected by a transvenous pacing lead? Pacing Clin Electrophysiol, 1996, 19:1682-1688.

[7] Rickards AF, Bombardini T, Corbucci G, Plicchi G, for the Multicenter PEA Study Group. An implantable intracardiac accelerometer for monitoring myocardial contractility. Pacing Clin Electrophysiol, 1996, 19:2066-2071.

[8] Plicchi G, Marcelli E, Parlapiano M, Bombardini T: PEA I and PEA II based implantable hemodynamic monitor: Pre Clinical studies in sheep. Europe, 2002, 4:49-54.

[9] Bordachar P, Garrigue S, Ritter P, Ploux S, Labrousse L, Casset C, Haissaguerre M, Dos Santos P: Contributions of a hemodynamic sensor embedded in an atrial lead in a porcine model. J Cardiovasc Electrophysiol, 2011, 22:579-583.

[10] Gras D, Kubler L, Ritter P, Anselme F, Delnoy PP, Bordachar P, Renesto F, Mabo P: Recording of Peak Endocardial Acceleration in the atrium. PACE, 2009, 32:S240-S246.

[11] Gleason WL, Braunwald E. Studies on the first derivative of the left ventricular pressure pulse in man. J Clin Invest, 1962, 41:80-84.

[12] Mason DT. Usefulness and limitations of the rate of rise of intraventricular pressure $(\mathrm{dP} / \mathrm{dt})$ in the evolution of myocardial contractility in man. Am J Cardiol, 1969, 23:516-527.

[13] Tassin A, Kobeissi A, Vitali L, Rouleau F, Ritter P, Gaggini G, Dupuis JM. Relationship between amplitude and timing of hearts sounds and endocardial acceleration. Pacin Clin Electrophysiol, 2009, 32 (Suppl.1):S101-S104.

[14] Clementy J, Kobeissi A, Garrigue S, Jaïs P, Le Metayer P, Haïssaguerre M. Validation by serial standardized testing of a new rate-responsive pacemaker sensor based on variations in myocardial contractility. Europace, 2001, 3:124-131.

[15] Clementy J, Garrigue S, Le Mouroux A, Gaggini G, on behalf of the European PEA Clinical Investigation Group. Dual-chamber rate responsive pacing system driven by contractility: short-term study and final assessment after one-year follow-up. Progress in Clinical Pacing, 1998, Proceedings Book; Chapter 33:219-224.

[16] Greco EM, Ferrario M, Romano S. Clinical evaluation of peak endocardial acceleration as a sensor for rate-responsive pacing. PACE, 2003, 26(Pt 1):812-818.

[17] Kasinski D, Grubb BP, Temesy-Armos P. Pathological aspects of neurocardiogenic syncope: current concepts and new perspectives. Pacing Clin Electrophysiol, 1995, 18:716-24.

[18] Deharo JC, Peyre JP, Chalvidan T, Thirion X, Valli M, Ritter P, Djiane P. Continuous monitoring of an endocardial index of myocardial contractility during head-up tilt test. Am Heart J, 2000, 139(6):1022-1030.

[19] Mangin L, Kobeissi A, Lelouche D, Dhérouville TY, Mansier P, Swynghedauw B, Macquin-Mavier I. Simultaneous analysis of heart rate variability and myocardial contractility during head-up tilt in patients with vasovagal syncope. J Cardiovasc Electrophysiol, 2001, 12(6):639-644.

[20] Deharo JC, Borri-Brunetto A, Bellocci F, Barbonaglia L, Occhetta E, Fasciolo L, Bocchiardo M, Rognoni G. DDDR pacing driven by contractility versus DDI pacing in vasovagal syncope: a multicenter, randomized study. Pacing Clin Electrophysiol, 2003, 26(1 Pt 2):447-550.

[21] Ritter P, Padeletti L, Gillio-Meina L, Gaggini G. Determination of the optimal atrioventricular delay in DDD pacing Comparison between echo and peak endocardial acceleration measurements. Europace, 1999, 1:126-130.

[22] Leung SK, Lau CP, Lam CT, Ho S, Tse HF, Yu CM, Lee K, Tang MO, To KM, Renesto F. Automatic optimization of resting and exercise atrioventricular interval using a peak endocardial acceleration 
sensor: validation with Doppler echocardiography and direct cardiac output measurements. Pacing Clin Electrophysiol, 2000, 23(11 Pt 2):1762-1766.

[23] Cuoco FA, Gold MR. Optimization of cardiac resynchronization therapy: importance of programmed parameters. J Cardiovasc Electrophysiol, 2012, 23(1):110-118.

[24] Mullens W, Grimm RA, Verga T, Dresing T, Starling RC, Wilkoff BL, Tang WH. Insights from a cardiac resynchronization optimization clinic as part of a heart failure disease management program. $J$ Am Coll Cardiol, 2009, 53(9):765-773.

[25] Delnoy PP, Marcelli E, Oudeluttikhuis H, Nicastia D, Renesto F, Cercenelli L, Plicchi G. Validation of a peak endocardial acceleration-based algorithm to optimize cardiac resynchronization: early clinical results. Europace, 2008, 10(7):801-808.

[26] Dupuis JM, Kobeissi A, Vitali L, Gaggini G, Mehreb M, Rouleau F, Lefthériotis G, Ritter P, Victor J. Programming optimal atrioventricular delay in dual chamber pacing using Endocardial Acceleration: comparison with a standard echocardiographic procedure. PACE, 2003, 26[Pt. II]:210-213.

[27] Ritter P, Delnoy PP, Padeletti L, Lunati M, Naegele H, Borri-Brunetto A, Silvestre J. A randomized pilot study of optimization of cardiac resynchronization therapy in sinus rhythm patients using a peak endocardial acceleration sensor vs. standard methods. Europace, 2012, 14(9):1324-1333.

[28] RESPOND-CRT, clinicaltrials.gov id: NCT01534234. www.clinicaltrials.gov.

[29] Donal E, Giorgis L, Cazeau S, Leclercq C, Senhadji L, Amblard A, Jauvert G, Burban M, Hernandez A, Mabo P. Endocardial acceleration (sonR) vs. ultrasound-derived time intervals in recipients of cardiac resynchronization therapy system. Europace, 2011, 13 (3):402-8.

[30] Bertini M, Delgrado V, Bax JJ, Van de Veire NRL. Why, how and when do we need to optimize the setting of cardiac resynchronization therapy?. Europace, 2009, 11:v46-v57.

[31] Ellenbogen KA, Gold MR, Meyer TE, Fernndez Lozano I, Mittal S, Waggoner AD, Lemke B, Singh JP, Spinale FG, Van Eyk JE, Whitehill J, Weiner S, Bedi M, Rapkin J, Stein KM. Primary results from the SmartDelay determined AV optimization: a comparison to other AV delay methods used in cardiac resynchronization therapy (SMART-AV) trial: a randomized trial comparing empirical, echocardiography-guided, and algorithmic atrioventricular delay programming in cardiac resynchronization therapy. Circulation, 2010, 21;122(25):2660-8.

[32] Abraham WT, Gras D, Yu CM, Guzzo L, Gupta MS; FREEDOM Steering Committee. Rationale and design of a randomized clinical trial to assess the safety and efficacy of frequent optimization of cardiac resynchronization therapy: the Frequent Optimization Study Using the QuickOpt Method (FREEDOM) trial. Am Heart J, 2010, 159(6):944-948.

[33] Porciani MC, Ricceri I, Attanà P, Pennesi M, michelotti F, Mascia G, Sacchi S, Hashtroudi L, Padeletti L. Discordant electrical and mechanical atrial delays affect intracavitary electrograms-based cardiac resynchronization therapy optimization. Europace, 2012, 14(4):593-8. 


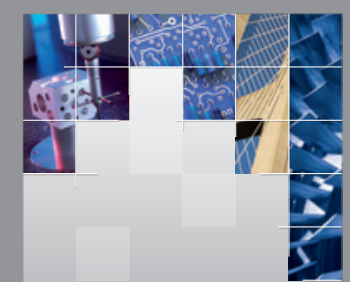

\section{Enfincering}
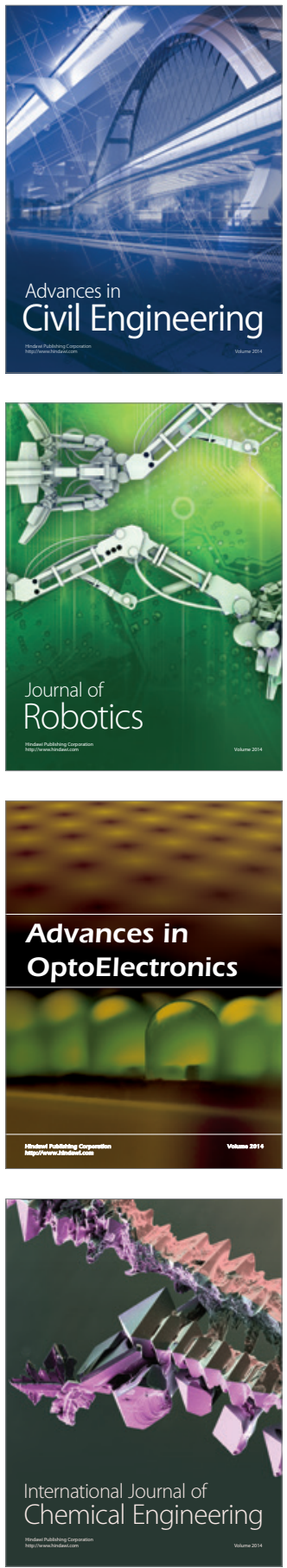

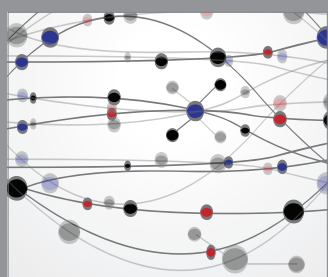

The Scientific World Journal

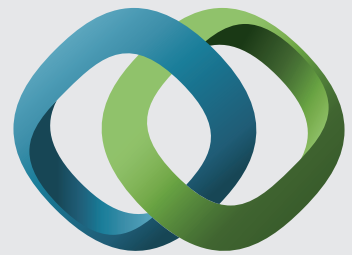

\section{Hindawi}

Submit your manuscripts at

http://www.hindawi.com
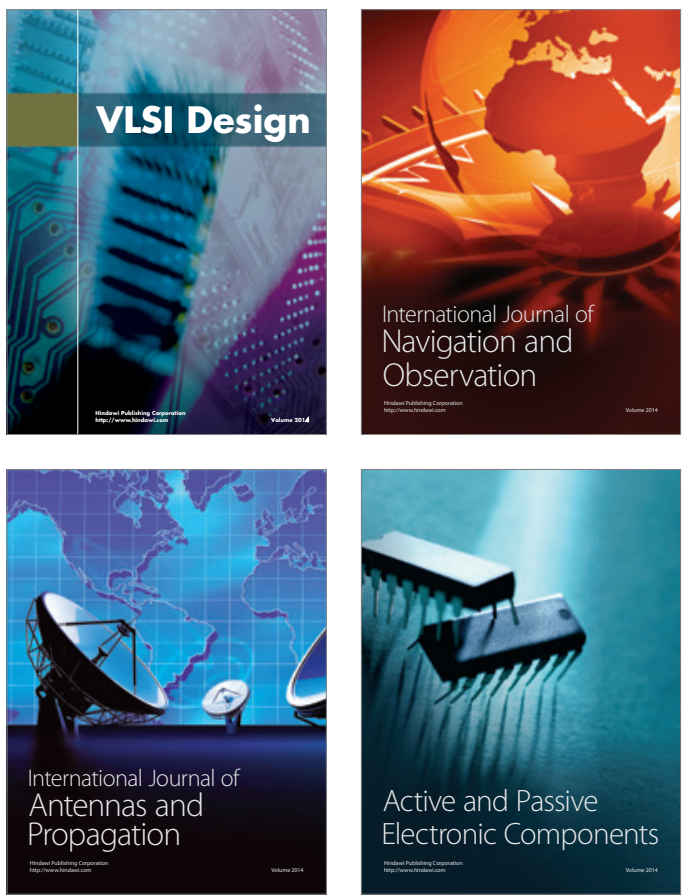
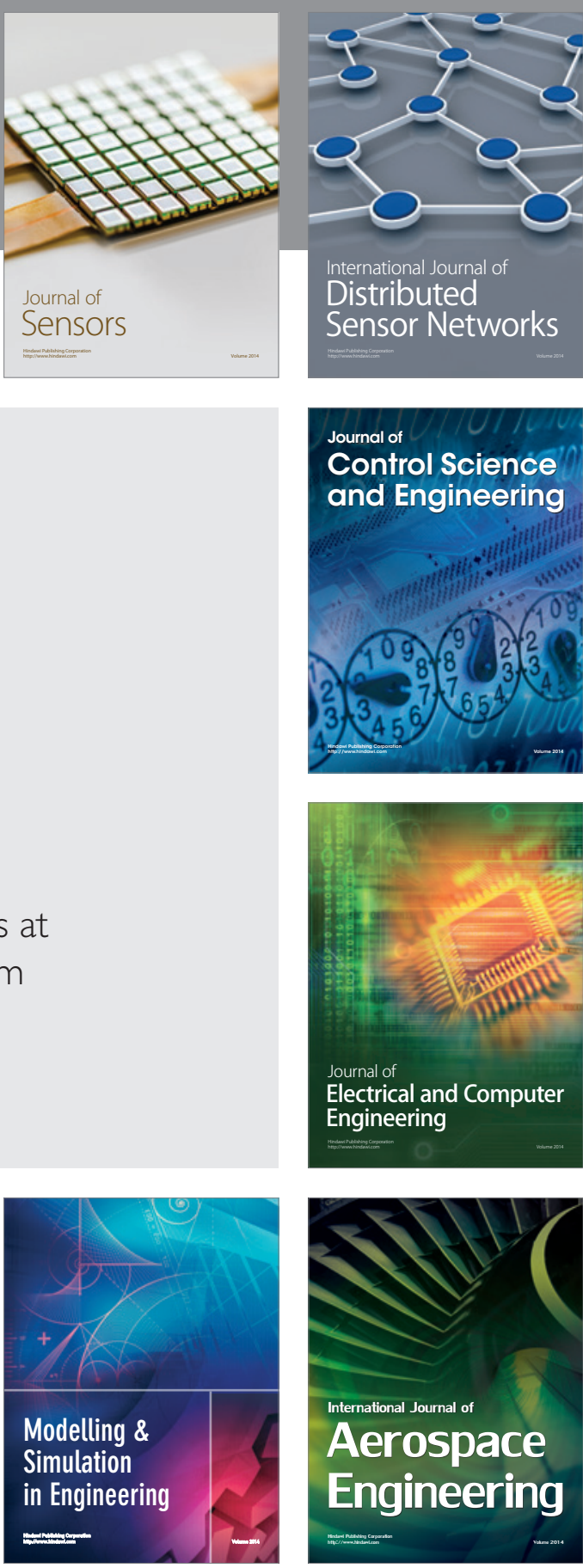

International Journal of

Distributed

Sensor Networks

Journal of

Control Science

and Engineering
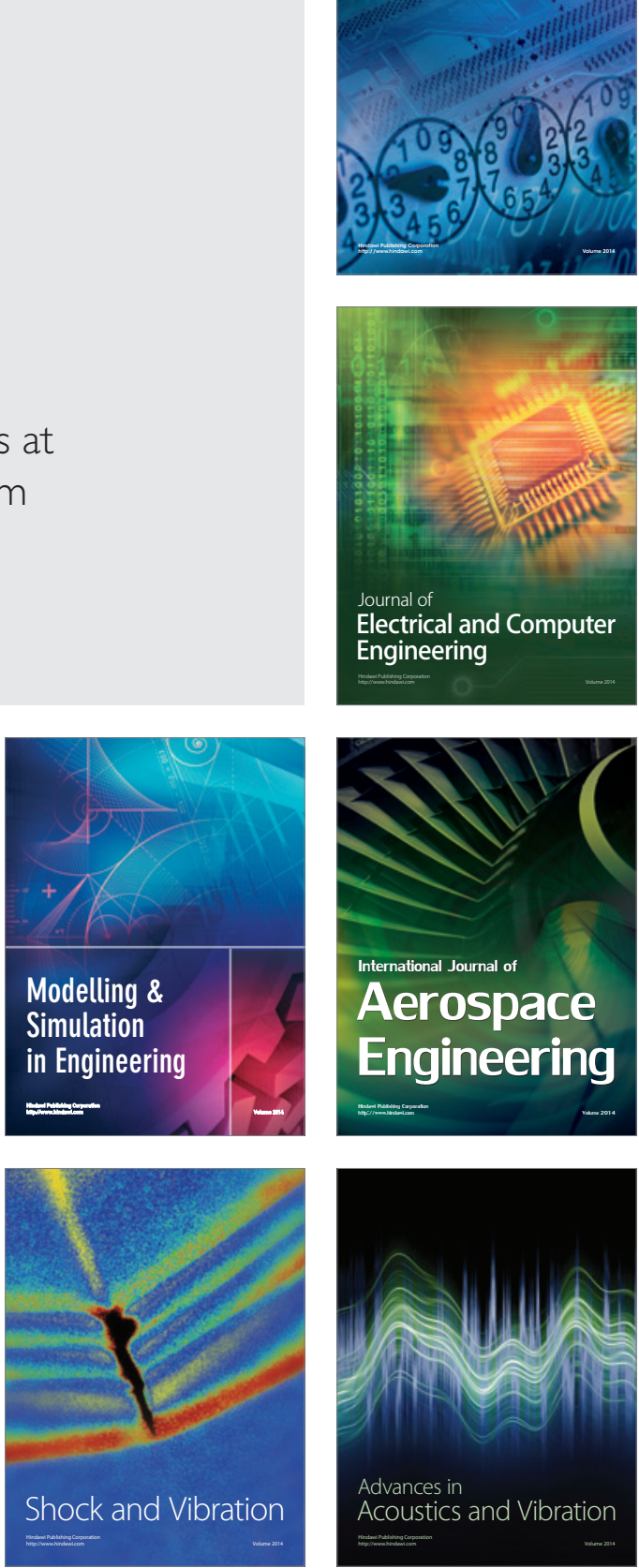